\title{
INVESTIGACIONES
}

\section{Innovar en el aula. Contradicciones entre nuevas herramientas y viejos roles como medio para trasformar la práctica*}

\author{
Innovation in the classroom. Contradictions between new tools \\ and old roles as a means to transform the teaching practice
}

\author{
Macarena Lamas, ${ }^{a}$ José-Luis Lalueza ${ }^{b}$ \\ ${ }^{a}$ Universidad San Sebastián \\ Telf.:56-63-2632832. Correo electrónico: macarena.lamas@uss.cl \\ ${ }^{\mathrm{b}}$ Universidad Autónoma de Barcelona \\ Telf.: 34-93-5811377. Correo electrónico: joseluis.lalueza@uab.cat
}

\section{RESUMEN}

El artículo expone una investigación cualitativa, longitudinal que utiliza la metodología de investigación-acción acerca del desarrollo de una actividad innovadora en una escuela ubicada en un contexto vulnerable y de riesgo de exclusión social. Nuestro propósito es contribuir a la reflexión de lo que sucede en estos entornos cuando se adopta una iniciativa que lleva al desarrollo conjunto de un proyecto de innovación educativa, así como conocer los factores que favorecen o interfieren en la apropiación de tal innovación. Nuestro marco teórico toma los planteamientos del enfoque sistémico y de la Teoría de la Actividad Histórico-Cultural. Nuestros resultados indican la emergencia de contradicciones entre las prácticas, herramientas y metas del sistema de actividad innovador y el sistema de actividad de aula, que se convierten en el motor del cambio a través de la búsqueda colectiva de resolución de estas contradicciones y construcción de nuevos roles en la actividad innovadora.

Palabras clave: innovación escolar, cambio de rol, práctica de maestros, sistema de actividad, contradicciones.

\begin{abstract}
The paper reports on a longitudinal action research project in which a team of university-based researchers work with low-income, minority, and immigrant children to improve academic skills through technology-based activities. Our focus is on the response of the school system to innovations that disturb the status quo. We work within a theoretical framework constructed from systems theory and cultural-historical activity theory. We discovered that the innovation resulted in the emergence of contradictions between classroom activities and norms established within the conventional goals of the schools. These prompted collective responses by school personnel to resolve contradictions and develop new roles and pedagogical practices.

Key words: school innovation, changing roles, practicing teachers, system activity, contradictions.
\end{abstract}


Estudios Pedagógicos XLII, N 3: 243-258, 2016

INNOVAR EN EL AULA. CONTRADICCIONES ENTRE NUEVAS HERRAMIENTAS Y VIEJOS ROLES COMO MEDIO PARA TRASFORMAR LA PRÁCTICA

\section{INTRODUCCIÓN}

La innovación en educación escolar para la mejora de la práctica educativa es un tema ampliamente tratado en la literatura especializada, pero no por ello resuelto. Hasta la fecha, numerosas investigaciones se han orientado principalmente hacia la constatación del impacto y el desarrollo de actividades innovadoras, dejando tras de sí una extensa lista de consideraciones y requisitos a tener en cuenta antes de implementar el cambio planeado: ¿cómo actuar y qué hacer?, ¿qué aspectos didácticos u organizativos se deben modificar?, ¿qué aspectos ha de trasformar un centro para ser de calidad? Todas cuestiones prescriptivas que muestran una preocupación más práctica que teórica. Sin embargo, poco se ha atendido a lo que, en definitiva, ocurre en los centros escolares y para qué ocurre, a lo que sucede en el ámbito social, relacional y emocional de los maestros que desarrollan estas actividades, o al papel que cumple la historia del centro escolar en el desarrollo de los cambios y la cultura organizacional como elemento constituyente de las dinámicas de la escuela (Clarke, 2006; Fullan, 2002; Murillo, 2003).

Nuestro trabajo pretende indagar en la experiencia de un grupo de maestros que se involucran en el desarrollo e implementación de una actividad innovadora de aula en su escuela. Nos interesa conocer la dinámica del cambio, explorándolo desde el punto de vista de quienes participan en el sistema de actividad innovador. Será una aproximación desde la teoría de la actividad (Engeström, 2001, 2009; Leontiev, 1979; Vygotsky, 1979) y los aportes del enfoque sistémico (Bateson, 2000) en un entorno escolar multicultural donde confluyen una diversidad de metas educativas familiares, culturales y sociales que tienen un denominador común, los niños y niñas que asisten a clases.

\section{CAMBIO, APRENDIZAJE Y DESARROLLO EXPANSIVO}

La idea de cambio sobre el que se sustenta este trabajo está ligada tanto a las características sistémicas de las instituciones e individuos que las forman, como a su condición de entidades con capacidad para el aprendizaje y el desarrollo. Desde la Psicología Cultural, aprendizaje, cambio y desarrollo formarían una triada indisoluble que solamente puede ser comprendida a la luz del contexto social, cultural e histórico donde se configuran las prácticas socioculturales de la propia comunidad, la cual también se halla en proceso de cambio (Cole, 2011; Rogoff, 2003). Los miembros más jóvenes de cada comunidad son introducidos en prácticas clave para su desarrollo a través de un proceso guiado que involucra la participación en actividades colectivas, dirigidas hacia metas identificables por la comunidad, mediadas por herramientas u otros seres humanos (Leontiev, 1979; Rogoff, 1993). Desde esta definición entendemos que el grado de profundidad de los aprendizajes y de la apropiación de las prácticas está en relación a la forma en cómo se organiza la actividad colectiva, que permite crear el sentido de pertenencia y desarrollar una identidad como miembro de una comunidad de práctica, (Lave y Wenger, 1991), los aprendices transitan desde una participación periférica legítima (con un bajo nivel de apropiación de las prácticas y herramientas) hacia una participación central en las actividades socialmente establecidas.

La acción humana mediada y dirigida a metas toma la forma de sistemas de actividad complejos, organizados según unas determinadas normas y reglas de funcionamiento, que involucran división del trabajo y adscripción dentro de una comunidad más amplia donde se 
congregan otros sistemas de actividades diferentes, iguales o complementarios (Engeströn, 2001, 2009). En esta línea, Michael Cole (1999, 2011) llama la atención sobre el fenómeno de la coexistencia de diversos sistemas de actividades culturales o, como Hedegaard (2009) señala, de distintos tipos de participaciones adscritos a distintas prácticas, donde la escuela es parte de uno de estos espacios donde confluye la diversidad entre varios contextos de desarrollo de las personas. Ahora bien, en las escuelas estatales la práctica de los maestros y los artefactos que las median responden a metas educativas plasmadas en el currículum y una serie de serie de normas de funcionamiento, configurando así una red de supuestos que sustentan todo el sistema. Estas son las "restricciones socioinstitucionales" (Bateson, 2000) que delimitan los movimientos de los sujetos en un mapa trazado a través de la historia de la organización y sostenido a través de la institucionalización de la práctica. Pero, ¿qué sucede cuando estas metas educativas no son compartidas por las familias de los niños pertenecientes a distintos nichos culturales? ¿Cómo se debe pensar la enseñanza en las aulas multiculturales? Diversos análisis etnográficos de la práctica en el aula muestran grandes dificultades e inadecuaciones en las dinámicas de la institución escolar para la inclusión de los miembros de grupos minoritarios (Bereményi, 2011; Crespo et al., 2012; Fernández-Enguita, 1994).

Engeström, aplicando los principios de la teoría de la actividad en sus investigaciones acerca de las instituciones, ha desarrollado un sugerente trabajo sobre la capacidad de toda organización para desarrollar nuevos aprendizajes y provocar, por tanto, transformaciones expansivas en su sistema de forma deliberada (Engeström, 2001; Engeström y Sanino, 2010; Engeström et al., 2003; Igira y Aanestad, 2009). Señala que cuando una organización adopta un nuevo elemento externo (por ejemplo: una nueva tecnología, un nuevo procedimiento, la inmigración) se generan agravantes, conflictos y perturbaciones con los viejos elementos del sistema, (confrontación con las reglas, las normas de funcionamiento, la división del trabajo). Estas perturbaciones, entendidas como contradicciones entre lo establecido y lo nuevo, contienen una gran potencialidad para generar transformaciones sustantivas en las prácticas, ya que exigen a las personas elaborar una reconceptualización de su objeto de trabajo y del motivo de su actividad, un tercer objeto de la práctica que no se reduce a la dinámica entre objetos que compiten, se combinan o se complementan. Se trata de la co-creación colectiva de algo nuevo, nuevos objetos de la práctica, nuevas formas de funcionamiento que impulsan al sistema a una nueva fase de desarrollo, lo que se denomina ciclo de aprendizaje expansivo (Engeström, 2001, 2007, 2009; Engeströn et al. 2003). Lo que se pretende lograr en el caso que nos ocupa es el estudio de este ciclo donde se generan conflictos secundarios ante la llegada masiva del alumnado multicultural a las aulas y a la vez la introducción de un sistema de actividad innovador que pretende ser un aporte para su inclusión.

\section{PROYECTO SHERE ROM COMO PRÁCTICA INNOVADORA PARA LA INTEGRACIÓN DEL ALUMNADO MULTICULTURAL}

La actividad de innovación a la que hacemos referencia se basa en un modelo de práctica colaborativa para la inclusión social, la Fifth Dimension (5D), desarrollada por Michael Cole desde hace poco más de cuatro décadas en el Laboratory of Comparative Human Cognition, en la Universidad de California. Surge como proyecto prototípico para el desarrollo de 
sistemas de actividades basados en el aprendizaje colaborativo, mediados por las TIC y organizados en forma de cooperación entre comunidad y universidad, que actualmente se desarrolla a través de una red de investigación en universidades de Estados Unidos, Europa y América Latina (Cole y The Distributed Literacy Consortium, 2006; Nilsson y Nocon, 2005; Padrós et al., 2014a). En España, el modelo se representa en el proyecto conocido como "la casa de Shere Rom", que actualmente incluye ocho espacios, en tres escuelas de enseñanza primaria, un instituto de secundaria, dos locales gestionados por comunidades gitanas y dos centros cívicos de barrio (Luque y Lalueza, 2013; Padrós et al., 2014b).

Descrita en pocas palabras, la actividad del proyecto Shere Rom consiste en la colaboración de un grupo de niños y niñas que junto a estudiantes de la universidad resuelven tareas o misiones designadas por un personaje virtual, utilizando para ello diversas herramientas TIC. La actividad está orientada a la creación de pequeños relatos digitales con el fin de brindar a los niños la oportunidad de ser creadores y participantes activos en todo momento. El proceso transcurre en fases: planificación del trabajo a realizar, selección del tema, elaboración del guion, grabaciones, edición y finalmente visualización pública. Pero este proceso no es lineal, cada visualización lleva a una revisión crítica de lo hecho y a una reflexión para la toma de decisiones que en ocasiones lleva a los participantes a regresar sobre sus pasos hacia fases anteriores del proceso. La distribución del trabajo y las normas de la actividad no son definidas a priori, son fijadas durante el transcurso de la actividad por los participantes para su óptimo funcionamiento, los roles al interior del sistema de actividad se construyen potenciándose la rotación donde una misma persona que puede ocupar un nivel de experto y enseñar, y en otra ocasión puede aprender de otros.

El modelo instruccional del proyecto se basa en la participación dentro de una comunidad de práctica compuesta por niños, estudiantes, maestros, una asesora externa y la comunidad más amplia representada por familiares de los niños que en ocasiones tienen una participación periférica. Los niños aprenden cuando colaboran unos con otros y crean historias conectadas directamente con las prácticas habituales de su comunidad o con sus propios intereses. La meta de la actividad es explícita, los objetivos y contenidos de cada relato digital se negocian entre todos los miembros, desarrollando una narración compartida.

\section{METODOLOGÍA}

El objetivo de la investigación que presentamos en este artículo consiste en analizar y describir los cambios producidos en la práctica educativa de un grupo de maestras ${ }^{1}$ que participan de una actividad innovadora, tal y como ocurre en un escenario de aula en un centro educativo multicultural. Nos interesa saber: a) qué ocurre en los aspectos vivenciales, conductuales, emocionales de las maestras que desarrollan la actividad, b) qué factores favorecen o entorpecen el ciclo de aprendizaje expansivo de la actividad, c) cómo opera el proceso de apropiación de las herramientas y metas.

Este estudio se enmarca en un programa de investigación-acción, de modo que la actividad específica que constituye nuestra unidad de análisis, también es explorada por las

A partir de ahora utilizaremos el genérico "maestras" o "tutoras", ya que en su gran mayoría las participantes en este estudio han sido mujeres, además utilizaremos el genérico "niños" para referirnos tanto a hombres como a mujeres. 
maestras como agentes activas en la reflexión, diseño, planificación, ejecución, evaluación y vuelta a la reflexión sobre las acciones, cumpliendo un ciclo en espiral cuya finalidad es la mejora de su práctica. Se comienza por la introducción de la actividad innovadora en una de las escuelas de primaria que participan del proyecto Shere Rom. Esta implementación es discutida y negociada previamente con los miembros del equipo directivo del centro educativo, fijándose acuerdos y co-responsabilidades para su ejecución; así, la escuela proveerá de herramientas, espacios e infraestructura, como de una maestra que tomará el rol de coordinadora de la actividad, mientras el equipo de investigadores facilitará algunas herramientas informáticas (software y plataforma virtual), además de los estudiantes universitarios que darán soporte a la actividad. Se cuenta con una asesora externa al centro (investigadora) quien acompaña a los maestros en el desarrollo de la iniciativa.

Para posibilitar la profundidad en la indagación del proceso de cambio, optamos por un estudio de caso longitudinal que abarca cuatro cursos académicos en una escuela pública de la provincia de Barcelona.

Se utilizaron tres técnicas principales de recolección de datos: entrevistas, taller de investigación-acción y observación participante. Las primeras consistieron en una entrevista semiestructurada a la primera asesora de la actividad (anterior a la investigadora de este estudio), y una entrevista grupal con 4 maestras y un maestro que desarrollan la actividad en la hora de informática, ambas entrevistas fueron realizadas una vez finalizado el primer año de estudio. Se realizaron 12 talleres de diseño con la participación del equipo de maestras y la actual asesora externa (3 anuales: planificación, desarrollo y evaluación de la actividad). Por último, nos servimos de la toma pormenorizada de notas de campo del día a día de las actividades durante los cuatro años que dura la investigación.

\subsection{PROCEDIMIENTO}

La anterior asesora externa llevaba un registro de su experiencia en un diario de campo que comentaba periódicamente con el grupo de investigadores. A partir de estos reportes se confeccionó el temario para la entrevista que sirviera como lista de chequeo y así controlar el abordaje de todos los aspectos necesarios para obtener un relato del desarrollo histórico de la actividad. La entrevista adopta una forma narrativa al estilo de las historias de vida, cuya duración fue de una hora y media. A partir de los contenidos de esta entrevista se confeccionó el temario para la entrevista grupal con maestras, cuyo formato fue próximo al de un grupo de discusión, pero las llamamos entrevistas grupales debido al carácter directivo de las entrevistadoras. Participaron cuatro maestras (dos tutoras de $5^{\circ}$, una de $4^{\circ}$ y la directora de la escuela) y un maestro (tutor de $4^{\circ}$ ), el encuentro duró aproximadamente dos horas.

Las entrevistas fueron grabadas y transcritas, para posteriormente ser analizadas a través del software de análisis de textos ATLAS.ti (Muñoz, 2004; Saldaña, 2009; Hwang, 2008). Tras la creación de una herramienta hermenéutica que contenía ambas entrevistas, se realizó una codificación sistemática de las citas en paralelo por tres investigadores que posteriormente debieron consensuar tal segmentación. No se partió de una categorización previa de análisis, sino de categorías que surgen de los datos siguiendo el modelo de la Grounded Theory (Pidgeon y Henwood, 1997). Mediante la triangulación de esta información, se obtiene un primer listado común de códigos. Este listado de códigos se agrupó posteriormente en tres familias de categorías, que a su vez contienen códigos de primer nivel como se muestra en la siguiente tabla: 
Tabla 1. Familias de categorías y códigos. Instrumento entrevistas

\begin{tabular}{|c|c|c|c|}
\hline Familia & Definición Operacional & Categoría & Código de primer nivel \\
\hline \multirow[b]{2}{*}{ Restricciones } & \multirow{2}{*}{$\begin{array}{l}\text { Características de la escuela } \\
\text { vista como sistema, que } \\
\text { involucra aspectos } \\
\text { contextuales de la comunidad } \\
\text { donde se inserta } \\
\text { (características del entorno } \\
\text { socioeconómico y } \\
\text { socioeducativo del alumnado } \\
\text { y sus familias). Patrones de } \\
\text { acción que dan forma al } \\
\text { sistema, a su identidad y que } \\
\text { guían su funcionamiento y } \\
\text { respuestas frente a la novedad. }\end{array}$} & $\begin{array}{l}\text { Factores } \\
\text { contextuales } \\
(\text { FC) }\end{array}$ & $\begin{array}{l}\text { FC_alumno académico } \\
\text { FC_alumno social } \\
\text { FC_familia } \\
\text { FC_cultural }\end{array}$ \\
\hline & & $\begin{array}{l}\text { Cultura escolar } \\
\text { (CE) }\end{array}$ & $\begin{array}{l}\text { CE_disciplina } \\
\text { CE_relaciones de poder } \\
\text { CE_atención a la diversidad } \\
\text { CE_liderazgo directivo } \\
\text { CE_resistencias } \\
\text { CE_condicionantes }\end{array}$ \\
\hline \multirow{3}{*}{ Cambios } & \multirow{3}{*}{$\begin{array}{l}\text { Cambios observados en los } \\
\text { niños y maestras que } \\
\text { participan en la actividad } \\
\text { innovadora. } \\
\text { Cambios observados en los } \\
\text { roles de participación } \\
\text { dirigidos hacia la } \\
\text { coordinación para la acción. }\end{array}$} & $\begin{array}{l}\text { Cambios } \\
\text { Alumnos (AL) }\end{array}$ & $\begin{array}{l}\text { AL_competencia } \\
\text { AL_TIC } \\
\text { AL_social } \\
\text { AL_motivación }\end{array}$ \\
\hline & & $\begin{array}{l}\text { Cambios } \\
\text { maestras }(\mathbf{C M})\end{array}$ & $\begin{array}{l}\text { CM_maestra } \\
\text { CM_coordinadora }\end{array}$ \\
\hline & & Objeto (CO) & $\begin{array}{l}\text { CO_roles } \\
\text { CO_organización } \\
\text { CO_negociación } \\
\text { CO_orientación al cambio }\end{array}$ \\
\hline $\begin{array}{l}\text { Modelo 5D } \\
\text { (MO) }\end{array}$ & $\begin{array}{l}\text { Uso y apropiación de las } \\
\text { principales herramientas de la } \\
\text { actividad innovadora (modelo } \\
\text { 5D). }\end{array}$ & $\begin{array}{l}\text { Herramientas } \\
\text { (HE) }\end{array}$ & $\begin{array}{l}\text { HE_TIC } \\
\text { HE_estudiantes } \\
\text { HE_Mago }\end{array}$ \\
\hline
\end{tabular}

Se realizó una aproximación etnográfica a la actividad, tomándose notas de campo exhaustivas y detalladas acerca de su desarrollo durante los 4 años, recogidas por dos miembros del equipo de investigadores.

Se consideran en este análisis todas las entrevistas informales con las tutoras y los talleres de diseño. Para el análisis de los datos etnográficos también se utilizó el software ATLAS.ti creando una unidad hermenéutica que contiene todos los datos. En primer lugar, tres investigadores trabajaron en solitario sobre el análisis del primer año etnográfico, siguiendo la estrategia de codificación down-top, a partir de los datos. Posteriormente, se pusieron en común los códigos creados hasta obtener un listado preliminar de categorías generales consensuadas para continuar del mismo modo con los demás datos etnográficos. En una segunda fase del análisis se vincularon códigos de segundo nivel de cada categoría, para posteriormente crear familias de categorías previas provenientes de nuestro marco teórico (familia sujetos; familia objeto; familia herramientas; distribución del trabajo, contradicciones), siguiendo una estrategia combinada de codificación bottom-up/topdown, como se muestra en la siguiente tabla: 
Tabla 2. Familias de categorías y códigos. Instrumento etnografías

\begin{tabular}{|c|c|c|c|}
\hline Familia & Definición Operacional & Categoría & Código de primer nivel \\
\hline \multirow{7}{*}{ Sujeto maestras } & \multirow{7}{*}{$\begin{array}{l}\text { Toda aquella información que } \\
\text { nos ayuda a perfilar el rol de la } \\
\text { maestra en la actividad } \\
\text { innovadora, en los aspectos } \\
\text { conductuales, emocionales, de } \\
\text { relación y que nos permita } \\
\text { revelar el modelo de enseñanza } \\
\text { que utilizan. }\end{array}$} & \multirow{5}{*}{ Rol } & guía \\
\hline & & & encuadre/normas \\
\hline & & & estilo jerárquico \\
\hline & & & disciplina \\
\hline & & & formación \\
\hline & & \multirow{2}{*}{ M. Emocional } & vínculo/motivación \\
\hline & & & conflicto/desgaste \\
\hline \multirow{4}{*}{ Sujeto niños } & \multirow{4}{*}{$\begin{array}{l}\text { Toda aquella información que } \\
\text { nos ayude a perfilar el rol de los } \\
\text { niños dentro de la actividad } \\
\text { innovadora, en cuanto a los } \\
\text { aspectos emocionales, } \\
\text { conductuales y de relación } \\
\text { promovidos en la actividad. }\end{array}$} & \multirow{2}{*}{ N. Emocional } & relación/vínculo \\
\hline & & & motivación \\
\hline & & \multirow[b]{2}{*}{ Conductual } & Autorreg./autonomía \\
\hline & & & dispersión \\
\hline \multirow{6}{*}{$\begin{array}{l}\text { Objeto/ } \\
\text { Modelo de } \\
\text { enseñanza- } \\
\text { aprendizaje }\end{array}$} & \multirow{6}{*}{$\begin{array}{l}\text { Plan más o menos estructurado } \\
\text { para orientar la enseñanza en el } \\
\text { aula y que incluye el } \\
\text { comportamiento como maestro, } \\
\text { la teoría subyacente y el clima } \\
\text { social. }\end{array}$} & \multirow{2}{*}{ Modelo } & participativa \\
\hline & & & transmisión \\
\hline & & \multirow{4}{*}{ Estrategias } & narratividad \\
\hline & & & negociación \\
\hline & & & tarea dirigida a metas \\
\hline & & & tarea desconectada \\
\hline \multirow{3}{*}{ Herramientas } & \multirow{3}{*}{$\begin{array}{l}\text { Principales herramientas que } \\
\text { median la actividad. }\end{array}$} & \multirow{3}{*}{ Mediadores } & TIC \\
\hline & & & estudiantes UAB \\
\hline & & & mago \\
\hline \multirow{4}{*}{$\begin{array}{l}\text { Distribución del } \\
\text { trabajo }\end{array}$} & \multirow{4}{*}{$\begin{array}{l}\text { Reparto de tareas entre } \\
\text { diferentes agentes que implica a } \\
\text { su vez distribución de poder, } \\
\text { responsabilidad y status. }\end{array}$} & \multirow{2}{*}{ Coordinación } & coordinación maestros \\
\hline & & & coordinación UAB \\
\hline & & \multirow{2}{*}{ Colaboración } & colaboración en el diseño \\
\hline & & & transversalidad \\
\hline \multirow{2}{*}{ Comunidad } & \multirow{2}{*}{$\begin{array}{l}\text { Influencia de la diversidad } \\
\text { cultural en la actividad y de } \\
\text { otros agentes externos a esta. }\end{array}$} & & diversidad cultural \\
\hline & & & otros agentes \\
\hline \multirow{4}{*}{ Contradicciones } & \multirow{4}{*}{$\begin{array}{l}\text { Todo aquello que nos interpela, } \\
\text { que nos coloca en una situación } \\
\text { de encrucijada, de doble vínculo } \\
\text { o demandas contradictorias que } \\
\text { provocan un cierto malestar } \\
\text { vivencial o sensación de } \\
\text { "callejón sin salida". }\end{array}$} & \multirow{4}{*}{ Conflictos } & entre maestros \\
\hline & & & maestra_niños \\
\hline & & & entre niños \\
\hline & & & relaciones de poder \\
\hline
\end{tabular}


Por último, se realiza la triangulación entre métodos complementarios y distintas fuentes de datos. Además, las conclusiones del estudio fueron sometidas a consideración de los informantes quienes confirmaron los hallazgos.

\section{ANÁLISIS DE RESULTADOS: DATOS ENTREVISTAS Y MATERIAL ETNOGRÁFICO}

La presentación de los resultados se organiza en torno a 3 ejes temáticos: 1) restricciones del sistema escuela; 2) cambios en los actores (niños, maestras); 3) contradicciones entre sistemas. Estos ejes se corresponden con nuestras categorías principales y permiten responder principalmente a las preguntas de nuestra investigación. Las demás categorías de análisis están integradas dentro de la discusión.

\subsection{RESTRICCIONES DEL SISTEMA ESCUELA}

En el análisis de la categoría restricciones, encontramos información de la percepción de las maestras sobre el perfil académico, sociocultural y familiar de sus alumnos. También nos proporciona un acercamiento a la cultura organizacional a través de los códigos referidos a relaciones de poder, normas implícitas y explícitas al interior de la organización y orientación al cambio. Esta información parcial, por cuanto se trata del discurso de las maestras, se confirma en el relato de la asesora externa.

estaban llegando los niños más indeseables, de alguna forma, indeseables en el sentido, niños con estructuras familiares más complicadas, niños con relación familia-escuela, que se tenía que tratar muy bien... una escuela, que también está muy cerrada a nivel familiar, mucha desconfianza con respecto a las familias y que esto genera también que la familia desconfíe de la escuela y como la familia después no viene cuando se la convoca, le da argumentos a la escuela y es una bola que se va haciendo grande". (Entr_asesora_UAB/A1).

En un principio, la diversidad cultural no parece que conlleve beneficio alguno, los alumnos presentan serias dificultades para aprender en las principales áreas académicas, se dan diversas formas de absentismo, baja motivación y elevado fracaso escolar que las entrevistadas asocian con una baja expectativa académica transgeneracional.

es que se nota diferencia entre la etnia gitana y el resto... Yo de mi clase, igual yo pienso que quizás los inmigrantes que vienen de Sudamérica o... o de Marruecos, quizás tienen más posibilidades de... hacer una carrera o... algo más con su futuro que los gitanos. Porque las niñas ya ves que... todo el mundo... yo pienso que todos admiran a sus padres, ¿no? Pero... las niñas, tienen como referente a su madre y a su padre, y las niñas lo que quieren es... las gitanas quieren ir al mercado, quieren tener niños, y quieren eso. No aspiran a nada más. (Entr._maestro_Jo_BS/A1).

La asesora externa describe explícitamente una estructura y unas relaciones muy jerarquizadas, de roles rígidos previamente establecidos. Las maestras, por su parte, destacan la disciplina y el orden como valores fundamentales que les permiten desarrollar su práctica educativa. 
yo creo que es un proyecto más de garantizar la educación obligatoria a todos, pero sin garantizar la calidad, es decir, es una preocupación excesiva en la asistencia, en el orden, en el mantener las cosas como deberían ser dentro de una escuela. Algo que llama mucho la atención cuando estás en la escuela es la obsesión por las filas, la obsesión por cuestiones muy formales [...] Están muy centrados en mantener esa escuela normal, que parezca una escuela normal... (Entr._asesora._UAB/A1).

El encuentro entre dos sistemas de actividad nunca es fácil, sobre todo si consideramos su característica autocorrectiva (Bateson, 2000), es decir, la tendencia a aislar la perturbación, a esconderla. En el caso del inicio del proyecto en la escuela, la frase del director es muy esclarecedora al respecto: "si a mí no me da trabajo, es en horario extraescolar y no molestáis"...bien y empezamos (asesora._UAB/A1).

Incluso cuando la actividad pasa a formar parte de una asignatura escolar, queda relegada a la clase de informática. La actividad dentro de la dinámica de la escuela es percibida como caótica, sin aparentes normas o reglas de funcionamiento.

lo que hacemos y desde los profesores que no han estado implicados en el proyecto, la visión que se tiene es que hacemos ruido y que están descontrolados y que están corriendo por el pasillo, de nuevo el orden es importante no, y esto era algo que también teníamos dificultades". (Entr._asesora._UAB/A1).

En resumen, de la exposición de los resultados obtenidos en la categoría restricciones, se destacan tres elementos relevantes: el primero, la existencia en la escuela de una población con determinadas características, que de lo expuesto por las maestras se asocia a familias en riesgo de exclusión social, pocos recursos socioeducativos, hándicap en los alumnos y alto nivel de diversidad cultural. En segundo lugar, las respuestas de la escuela frente a esta población se caracterizan por el intento de homogenizar a la población y respuestas de tipo compensatorio ante las dificultades en los alumnos para aprender. Y tercero, la llegada del proyecto a la escuela nos revela su dinámica interna, caracterizada por una estructura jerárquica, de normas y roles rígidos, donde la entrada del proyecto es vista como algo ajeno a dicha dinámica, quedando relegado a un espacio de actividad extraescolar.

\subsection{CAMBIOS EN EL SUJETO: MAESTRAS NIÑOS}

A través de los años que dura la investigación, podemos confirmar la cualidad del rol de las maestras como en permanente construcción. En un principio, las maestras novatas en la actividad se limitan a observar la dinámica que se desarrolla entre los niños y las estudiantes de la universidad, y a estar bajo la dirección y guía de la maestra coordinadora. El apoyo de los estudiantes universitarios les resulta clave para poder llevar a cabo la actividad, no únicamente por el control directo de los niños cuando ellas deben trabajar con un pequeño grupo, sino porque sirven de referente de actuación.

Esta construcción del rol no se presenta sin dificultades, las maestras, en varias ocasiones, manifiestan sentirse "perdidas en la actividad" y no saber muy bien cómo operar ante un rol que desconocen. Este periodo se caracteriza por un estado de confusión y ansiedad, observándose también un incremento en las acciones de coordinación y participación en el diseño. 
Estudios Pedagógicos XLII, $\mathrm{N}^{\circ}$ 3: 243-258, 2016

INNOVAR EN EL AULA. CONTRADICCIONES ENTRE NUEVAS HERRAMIENTAS Y VIEJOS ROLES COMO MEDIO PARA TRASFORMAR LA PRÁCTICA

$P i$, una de las maestras nuevas este año, dice no tener claridad sobre cuál es su rol dentro de la actividad, un rol concreto, y por lo tanto siente que no ha hecho nada. Yv, por su parte, dice que en un principio le sucedió lo mismo, se sentía perdida en la actividad y no sabía bien qué hacer, pero que poco a poco ha ido entendiendo cuál es su papel como guía, más como organizadora de la clase, ella señala que ha visto cómo el trabajo grupal ha enriquecido la interacción entre pares de su grupo clase. Los hábitos que se crean dentro de la actividad como repartir el trabajo, llegar a acuerdos a través de la negociación ayudan bastante a ello. (TD_BS_A3).

Con el paso del tiempo, las maestras van ganando en pericia para guiar y encuadrar la actividad, siguiendo un estilo de relación con sus alumnos más cercano a un modelo de participación donde lo afectivo emerge, destacando la mejora en la comunicación y un acercamiento más horizontal. Poco a poco prestan mayor interés en el desarrollo de la actividad más que en el resultado de la tarea. A su vez, manifiestan su interés por trasladar algunas actividades del proyecto a otras áreas del currículum.

Durante el desarrollo de las actividades, tanto Ma como Mi guían la sesión. Las dos utilizan estrategias como preguntar a los niños para hacerlos participar, pedir la atención de los compañeros, respeto hacia los demás y los niños responden bien. Ma establece contacto físico con los niños, los acaricia, les lleva hacia adelante para hablar, se mantiene a su lado cuando los niños más tímidos se presentan. Los niños siguen las indicaciones de Mi rápidamente, se nota mucha complicidad y cercanía entre esta maestra y sus alumnos. (Etn_BS_A3).

Los cambios en las maestras no se pueden entender al margen de los cambios producidos en los niños, es más bien la interrelación entre ambos lo que nos ofrece una explicación del cuadro completo. Las modificaciones que gradualmente van incorporando a su práctica se muestran como parte de un proceso sistémico y colectivo que no depende de la suma individual de lo aprendido. En los niños se observa una rápida adaptación a la dinámica de la actividad, ellos parecen estar desde un primer momento dispuestos, impacientes, motivados por comenzar las actividades y esperan ansiosos la llegada de estudiantes de la Universidad. En los pequeños grupos de trabajo se esfuerzan por explicarse las cosas unos a otros, se ayudan entre sí, despliegan destrezas en el trabajo grupal y en la resolución de conflictos.

Mientras que seguimos en redonda, se pasan un rato discutiendo el significado de la palabra "rilaora", que un niño ha pronunciado. En un momento dado, un niño ha dicho: "callaros, que M va a explicar el significado". A partir de ahí todos se han callado para escucharle. Y cuando ya parece haberlo explicado, una niña también gitana dice: "dejemos de discutir que ya es tarde hay que hacer el anuncio”. (Etn_BS_A3).

Tanto del análisis de entrevistas como de las notas de campo se observa cómo la rápida adquisición de competencias en el uso de las TIC infiere en ellos una mayor confianza en sus capacidades, lo que a su vez se relaciona a una mayor motivación en su uso.

La participación de los niños en la actividad se vuelve cada vez más autónoma: hacen preguntas, investigan, buscan, piden y otorgan información, siendo capaces de autorregular sus conductas y las de sus compañeros con bastante eficacia para poder alcanzar las metas específicas y compartidas del día a día. La actividad se muestra como un espacio propicio para la interacción, la colaboración y la negociación, impactando sobre todo en lo relacional y afectivo. 
[...] una de las niñas estaba muy preocupada y la incita a que me cuente el porqué. La niña estaba preocupada porque su compañero Bi, no quería trabajar en el grupo, pero hoy sí lo ha hecho, y muy bien, por lo que la misma niña lo felicita delante de todos nosotros y el niño se sonrie (Etn_BS_A4).

Ma comenta que antes del proyecto los niños de su clase casi ni se hablaban entre ellos, y si se hablaban era para insultarse. Cuenta también que una vez para una clase de lenguaje, intentaron con Mi, juntar ambos cursos y trabajar en conjunto, pero fue a su juicio imposible, muy caótico. Desde esa vez habían preferido no hacer más cosas en común, hasta que comenzaron a trabajar en el proyecto y ahora han visto muchos cambios, como por ejemplo que los niños se buscan entre ellos, se piden colaboraciones entre cursos, incluso se defienden unos a otros si algún niño de otro curso molesta a algún niño inmigrante. (Etn_BS_A4).

En la actividad predomina un modelo de enseñanza-aprendizaje basado en la colaboración y en la participación entre distintos actores (niños, estudiantes, maestras, investigadores, miembros de la comunidad, etc.), mediado por diversas herramientas y otros artefactos que cambian la configuración de la clase habitual, las relaciones y las formas de interacción. Ya en la actividad, las maestras van cediendo el control a los pequeños grupos de trabajo, en la medida que también se sienten más cómodas en la actividad, observan cambios positivos en sus alumnos y desarrollan nuevas funciones. Centran su labor en diversas tareas, como por ejemplo poner a disposición de los grupos las herramientas necesarias, asesorar en el uso de determinado software, mediar en conflictos, orientar las tareas de las estudiantes de la universidad. También se sitúan en el rol de aprendices junto a los niños, fomentando la ayuda mutua, la exploración y la autonomía. Es decir, vemos cómo a través de los años, se abren nuevas posibilidades de aprendizaje para ellas respecto a sus propios métodos de enseñanza (Mitton-Kükner, Nelson y Desrochers, 2010).

\begin{abstract}
El grupo que se encuentra a nuestro lado le pregunta a la tutora Mi si sabe cómo poder agregar efectos desde el estudio 10 (programa para edición de videos), Mi se acerca a ellos y dice: ¿ “qué es lo que queréis hacer?, explicadme", ella toma el mouse y junto con los niños y la estudiante comienzan a explorar el programa y las distintas posibilidades que da. (Etn_BS_A4).
\end{abstract}

\title{
5.3. CONTRADICCIONES ENTRE SISTEMAS
}

La incorporación del sistema de actividad innovador se observa como un proceso paulatino, lento y no exento de conflictos e incluso desgaste en los participantes. Hemos visto cómo la cultura escolar, a través de sus restricciones socio-institucionales, reacciona desde un primer momento frente a la innovación calificándola como caótica, fuera del control de los adultos, sin dirección aparente y en exceso permisiva para los niños. Esta idea, extendida entre los maestros que no participan directamente en la actividad, prevalece durante los años de investigación, ante ello las maestras del proyecto ven la necesidad de realizar mayores acciones de difusión.

Por otro lado, en ocasiones las maestras tienden a regresar a lo que conocen, a su tradicional forma de actuar en la escuela, sobre todo en los momentos de mayor dispersión de niños, desorden, descuido o uso inapropiado del material, imponiendo orden y disciplina a través de un estilo de relación jerárquica y vertical con sus alumnos. 
Estudios Pedagógicos XLII, $N^{\circ}$ 3: 243-258, 2016

INNOVAR EN EL AULA. CONTRADICCIONES ENTRE NUEVAS HERRAMIENTAS Y VIEJOS ROLES COMO MEDIO PARA TRASFORMAR LA PRÁCTICA

Luego, cuando ya se termina la sesión, Pa y Ma le piden a los niños que hagan una fila para subir al aula y estamos casi 10 minutos intentando que los niños hagan caso. En general, una vez que se da por terminada la sesión los niños se dispersan, gritan, juegan e incluso se pelean. Entonces Pa y Ma suben el tono de voz e imponiéndose, de esta manera logran que los niños hagan una la fila y salen así del aula. (Etn_BS_A3).

Hemos expuesto los primeros temores que aparecen en las maestras de reciente incorporación ante un rol que desconocen y ante una dinámica de aula que no manejan, por lo que no insistiremos nuevamente en ello. Sin embargo, podemos observar que aun cuando esta transición es óptima y se afianzan en su nuevo rol, la demanda de un trabajo coordinado, continuo y horizontal entre maestras atrae roces y tensión entre las mismas, asociado al encuentro entre las relaciones de poder jerarquizadas del sistema escuela y la necesaria distribución del trabajo del sistema de actividad innovador.

La coordinadora Ma dice no sentirse realmente una coordinadora, no puede tomar decisiones y cree que su punto de vista no es considerado ni tomado en cuenta por Pa, como ejemplo señala el trabajo que ha confeccionado para las jornadas de noviembre, que luego de mostrárselo a Pa, finalmente ella utilizará un power point que ya ha hecho. (RP_BS_A3).

\section{DISCUSIÓN Y CONCLUSIONES}

Para poder analizar y describir los cambios observados en la práctica educativa de este grupo de maestras en particular, no podemos abstraernos de lo que acontece en la práctica habitual del centro educativo. Esto, desde la percatación de las restricciones socioinstitucionales como la red de supuestos que sostienen el sistema y que nos muestran, por un lado, la respuesta del sistema escuela frente a la innovación, y por otro, las posibilidades de acción. Dichas restricciones que conforman su cultura escolar son, entre otros muchos elementos, el resultado de tradiciones, prácticas y ordenamientos jurídicos que definen los medios y fines de la institución escolar (Vila y Casares, 2009). Al mismo tiempo, nos encontramos con una cultura de centro que responde también a su historia particular, y nos permite acercarnos a la visión que se tiene acerca de los niños y las familias del sector o contexto comunitario. Estos aspectos influyen no solo en qué y cómo aprenden los niños en las aulas, sino también en lo que conlleva ser maestra en esa escuela y en cómo se aprende a serlo.

Como señala Bateson (2000), las restricciones no son fácilmente reconocibles como tales por las personas que conforman dicho sistema, ya que se integran en la forma de ser de la escuela; lo cotidiano, la rutina, los hábitos, los roles y funciones (Clarke, 2006) que le permiten conservar cierto equilibrio y estabilidad. Ante el cambio en la conformación de su alumnado (la llegada masiva de población multicultural), la escuela se resiste, activando lo que Maturana y Varela denominan "cambios de estado de un sistema con respecto a un medio al compensar las perturbaciones que recibe de éste" (Maturana y Varela, 1996, p. 92), reforzando sus defensas, es decir, sus patrones de acción arraigados e institucionalizados como forma de autocorregir la perturbación de origen externo. Así, durante el primer año de investigación son reiterados los desplazamientos de responsabilidad, las descalificaciones e, incluso, la culpabilización hacia lo externo, hacia las familias, por el fracaso escolar del alumnado, mientras que la dinámica interna de la escuela permanece incuestionable 
e incluso reforzada. Ha cambiado la población, los niños y sus familias son diferentes al alumnado más o menos homogéneo que se tenía antes, y como el sistema requiere volver a un cierto equilibrio, se introducen cambios que paradójicamente apuntan a mantener todo igual. Estos cambios de tipo 1 (Watzlawick, Weakland y Fisch, 1995) no afectan la identidad del sistema que en sí permanece invariable, por cuanto se refuerzan las medidas hacia el control y la uniformidad del alumnado.

Con la introducción del proyecto de innovación sucede más o menos lo mismo, no solamente por la tendencia evidente a desviar la actividad hacia una senda lateral, permitiendo en un principio su desarrollo solo fuera del horario escolar, sino también por su percepción como amenazante, caótico, fomentador del desorden, promotor de conductas poco deseables en los alumnos, resaltando su falta de estructura, límites y orden a juicio de esta cultura escolar. Incluso durante todos los años de investigación se puede apreciar que en los momentos de mayor dispersión de los niños, las maestras tienden a regresar a lo que conocen, a su tradicional forma de actuar, imponiendo el orden y la disciplina. Es decir, las restricciones socioinstitucionales que versan sobre estos valores fundamentales en el quehacer educativo, parecen estar grabadas a fuego en el rol educativo de las maestras.

Una vez dentro de la actividad, las maestras novatas comienzan a manifestar sus primeros temores y conflictos: inseguridad, el no saber hacer, sensación de no estar "haciendo nada", resistencias a establecer un tipo de relación horizontal con sus alumnos. Encontramos una explicación coherente para ello en la Teoría de la Actividad (Cole, 1999; Engeström, 2001, 2009; Leontiev, 1979). Cada sistema de actividad supone un particular tipo de relación entre sujeto y objeto y entre sujeto-sujeto, a través de herramientas mediadoras en el seno de una comunidad regulada por normas y organizada de acuerdo a un tipo de división del trabajo. En la actividad innovadora han cambiado las herramientas al introducir un particular uso de las TIC; se ha operado sobre la división del trabajo, redefiniendo los roles de los participantes; se ha variado la composición de la comunidad con la entrada de los estudiantes de la universidad y la asesora; se han cambiado las reglas abriendo un abanico de conductas pertinentes; se han redefinido los objetivos para hacerlos accesibles a los alumnos. Es decir, todos estos cambios en la organización del aula, suponen para las maestras un marcado contraste con la organización del aula tradicional. Ante ello, las dificultades para "encontrar su rol" dentro de la actividad innovadora no se hacen esperar y se manifiestan a través de conflictos y malestar, síntomas de las contradicciones existentes entre el rol que desempeñan en el aula habitual (que no tiene cabida en la nueva actividad) y el nuevo rol que se espera que desarrollen en la actividad innovadora, construido de manera activa por los participantes. Se requiere, como señala Fullan (2002), atender a la dinámica del cambio de rol que implica un cierto desaprendizaje y reaprendizaje en los sujetos que experimentan la incertidumbre ante la percatación de la escasa competencia para desempeñar nuevos papeles. Dicho de otro modo, las maestras y el maestro se encuentran ante una situación de doble vínculo (Watzlawick et al., 1995), donde no pueden operar en el nuevo sistema de actividad, utilizando las viejas prácticas y métodos de enseñanza del aula ordinaria, pero tampoco pueden maniobrar en un rol que desconocen y, es más, "no existe" todavía. Este estado vivencial de "callejón sin salida" les lleva a buscar soluciones colectivas para poder lidiar con las dificultades. Precisamente cuando las maestras señalan en las reuniones de taller su impotencia al no tener claridad sobre "el qué, ni el cómo", comienzan a diseñar juntos la actividad, a planificar las acciones del proyecto, o lo que en términos de Engeström y Sannino (2010) se traduce en ascender de lo abstracto y confuso 
Estudios Pedagógicos XLII, N 3: 243-258, 2016

INNOVAR EN EL AULA. CONTRADICCIONES ENTRE NUEVAS HERRAMIENTAS Y VIEJOS ROLES COMO MEDIO PARA TRASFORMAR LA PRÁCTICA

a algo concreto y tangible, aquí comienza a reconocerse un ciclo de aprendizaje colectivo y expansivo.

A medida que las maestras observan las interacciones entre estudiantes y niños, el tipo de relación horizontal que establecen y el uso que hacen de las herramientas, avanzan en la adquisición de estas y van comprendiendo también el potencial de su uso. Así, durante los últimos años de investigación, hemos visto cómo las maestras utilizan las TIC como medio para fomentar la exploración por parte de los niños, despertar su curiosidad y elevar la motivación en la clase. Pero ¿es la observación por sí sola suficiente para la apropiación en el uso de las herramientas y en la adquisición de las prácticas? Evidentemente, la apropiación abarca mucho más que eso, aun cuando observamos que, en un principio, es una acción necesaria y recurrente, también lo es el propiciar legitimidad a la participación de los actores (Lave y Wenger, 1991; Wenger, 2007; Wenger, McDermott y Snyder, 2002), sean maestras, niños, estudiantes universitarios. Clave para garantizar la legitimidad de la participación, es la estructura social dentro de la actividad, la forma en que se distribuye el trabajo y, por tanto, el poder; todos tienen un rol que cumplir del que depende también la posición de los demás. Hemos observado que la interacción entre los estudiantes universitarios y los niños se basa en una relación de tipo horizontal, los niños así se perciben como interlocutores válidos con capacidad para gestionar acciones y no solo cumplir tareas asignadas, por otra parte, las maestras nuevas en la actividad son guiadas por las maestras con más experiencia y por la coordinadora en la introducción a la nueva práctica. Así, las maestras se desplazan progresivamente desde una posición periférica legítima, a una posición central, en términos de Wenger (2001). Se trata, ahora, de tomar la iniciativa en el diseño de la actividad, la preparación y distribución de las herramientas, la presentación a los niños de la actividad, el ofrecimiento de ayuda personalizada, la dinamización del grupo, la mediación en situaciones de conflicto o confusión, la provisión de retroalimentación a las estudiantes, o la bienvenida a otros agentes de la comunidad que se prestan a ayudar. En definitiva, las maestras crean otros protocolos de acción para guiar la actividad, más adecuado a un modelo de enseñanza participativa y de aprendizaje colaborativo.

En conclusión, la actividad innovadora ha supuesto la incorporación en la escuela de nuevas restricciones, al menos para las maestras implicadas y participantes del proyecto. Este hecho se traduce en nuevas formas de operar, nuevos roles, nuevas normas de funcionamiento, nuevas formas de relacionarse con sus alumnos, y todo ello dirigido hacia la consecución de metas que no distan de las propias metas del quehacer educativo, el aprendizaje de los niños. Es decir, a través de la participación en un sistema de actividad diferente al tradicional de la escuela, han podido experimentar con otras posibilidades de acción educativa. Esta es una manera de actuar desde un punto de vista sistémico, la introducción de nuevas restricciones que amplíen las opciones disponibles de las maestras para el desarrollo de su práctica, que conduzcan a reconocer que otros caminos son posibles. Las contradicciones entre sistemas de actividad distintos las hemos visto reflejadas en los conflictos de rol, y a pesar de manifestarse en forma de tensión, se constituyen en el motor del cambio (Engeström, 2001, 2009; Engeström y Sanino, 2010) al confrontar a las maestras con una actividad que posee una serie de características (novedad, complejidad, desestructuración, ambigüedad, etc.), susceptible de activar en ellas la motivación intrínseca y, de este modo, provocar la necesidad de desarrollar una actividad exploratoria y una actividad de resolución colectiva dirigida a reducir el conflicto, la incertidumbre y la tensión generada por las características de la situación. 


\section{REFERENCIAS BIBLIOGRÁFICAS}

Bateson, G. (2000). Steps to an Ecology of Mind. Chicago: University of Chicago Press.

Bereményi, B. Á. (2011). Intercultural policies and teachers' contradictory views. The Roma case in Catalonian schools. Intercultural Education Journal, 22(5), 355-369.

Clarke, M.A. (2006). A Place To Stand: Essays for Educators in Troubled Times (4th ed.). Michigan: Ann Arbor, University of Michigan Press.

Cole, M. (1999). Psicología Cultural. Madrid: Morata.

Cole, M. (2011). Re-inventing Past Educational Practices for Future Pedagogical Success. Social Studies Journal, 40, 23-32.

Cole, M., \& The Distributed Literacy Consortium. (2006). The Fifth Dimension: An after-school program built on diversity. New York: Russell Sage.

Crespo, I., Rubio, R., López, C., \& Padrós, M. (2012). Lenguaje social, identidad e inclusión escolar en el discurso de los maestros. Cultura \& Educación, 24(2), 163-175.

Engeström,Y. (2001). Expansive Learning at Work: toward an activity theoretical reconceptualization Journal of Education at Work, 14(1), 133-156.

Engeström, Y. (2007). Enriching the Theory of Expansive Learning: Lessons From Journeys Toward Coconfiguration. Mind, Culture, and Activity, 14(1), 23-39.

Engeström, Y. (2009). From Learning Environments and Implementation to Activity Systems and Expansive Learning. An International Journal of Human Activity Theory, 2, 17-33.

Engeström, Y., Puonti, A., \& Seppänen, L. (2003). Spatial and temporal expansion of the object as a challenge for reorganizing work. In D. Nicolini, S. Gherardi y D. Yanow (Eds.), Knowing in organizations: A practice-based approach (pp. 151-186). Armonk: M. E. Sharpe.

Engeström, Y., \& Sannino, A. (2010). Studies of expansive learning: Foundations, Findings and future challenges. Educational Research Review, 5, 1-24.

Fernández-Enguita, M. (1994). Escuela y etnicidad: el caso del pueblo gitano. Madrid: CIDE.

Fullan, M. (2002). El significado del cambio educativo: Un cuarto de siglo de aprendizaje. Profesorado, Revista de currículum y formación del profesorado, 6(1), 1-14.

Hedegaard, M. (2009). “Children's development from a cultural-historical approach: children's activity in everyday local settings as foundation for their development". Mind Culture and Activity, 16(1), 149-168.

Hwang, S. (2008). Utilizing Qualitative Data Analysis Software: A Review of Atlas.ti. Social Science Computer Review, 26(4), 519-527.

Igira, F., \& Aanestad, M. (2009). Living with contradictions: Complementing Activity Theory with the Notion of "Installed Base" to Adress the Historical Dimension of Transformation. Mind, Culture, and Activity, 19(3), 209-333.

Lave, J., \& Wenger, E. (1991). Situated Learning. Legitimate peripherals participation (16a ed.). New York: Cambridge University Press.

Leontiev, A.N. (1979). The problem of activity in psychology. In J. V. Wertsch (Ed.), The concept of activity in Soviet psychology (pp. 37-71). Armonk, NY: Sharpe.

Luque, M. J., \& Lalueza, J.L. (2013). Aprendizaje colaborativo en comunidades de práctica en entornos de exclusión social. Un análisis de las interacciones. Revista de Educación, 362, $402-$ 428.

Maturana, H., \& Varela, F. (1996). El árbol del conocimiento. Madrid: Editorial Debate.

Mitton-Kükner, J., Nelson, C., \& Desrochers, C. (2010). "Narrative inquiry in service learning contexts: Possibilities forlearning about diversity in teacher education". Teaching and teacher education, 26, 1162-1169.

Muñoz, J. (2004). Análisis cualitativo de datos textuales con Atlas Ti 5. Barcelona: Universitat Autònoma de Barcelona.

Murillo, F.J. (2003). El movimiento teórico práctico de Mejora de la escuela. Algunas lecciones 
aprendidas para transformar los centros docentes. Revista Electrónica Iberoamericana sobre Calidad, Eficacia en Educación, 1(2). Recuperado de http://www.ice.deusto.es/RINACE/reice/ vol1n2/Murillo.pdf

Nilsson, M., \& Nocon, H. (2005). School of Tomorrow. Teaching and Technology in Local and Global Communities. Berna: Peter Lang.

Padrós, M., Sànchez-Busqués, S., Lalueza, J.L., \& Crespo, I. (2014a). The Shere Rom Project: Looking for Alternatives to the Educational Exclusion of Roma. International Journal for Research on Extended Education, 2014(2), 46-62.

Padrós, M., Sánchez-Busqués, S., Lalueza, J.L., Crespo, I., \& Lamas, M. (2014b). El proyecto Shere Rom. Fundamentos de una comunidad de prácticas para la inclusión educativa de grupos culturales minoritarios y en riesgo de exclusión social. Psicología, Conocimiento y Sociedad, $4(2), 138-162$.

Pidgeon, N., \& Henwood, K. (1997). Grounded theory: practical implementation. En J. Richardson (Ed.), Handbook of qualitative research methods for Psychology and the Social Sciences (pp. 86-101). Leicester: BPS Books.

Rogoff, B. (1993). Aprendices del pensamiento. Barcelona: Paidós.

Rogoff, B. (2003). The Cultural Nature of Human Development. Oxford NY: Oxford University Press.

Saldanã, J. (2009). The coding manual for qualitative researchers. Thousand Oaks Calif: Sage.

Vila, I., \& Casares, R. (2009). Educación y sociedad. Una perspectiva sobre las relaciones entre la escuela y el entorno social. Barcelona: Horsori.

Vygotsky, L.S. (1979). El desarrollo de los procesos psicológicos superiores. Barcelona: Editorial crítica.

Watzlawick, P., Weakland, J. H., \& Fisch, R. (1995). Cambio. Formación y solución de los problemas humanos (9a ed.). Barcelona: Herder.

Wenger, E. (2001). Comunidades de práctica. Aprendizaje, significado e Identidad. Cognición y Desarrollo Humano. Barcelona: Paidós.

Wenger, E. (2007). All communities of practice need to find their 'spirit', which can be called their learning companionship. EU Conference "eLearning Lisboa 2007”. Recuperado de http://www. elearningeuropa.info/directory/?page $=$ doc\&doc_id $=10476 \&$ doclng $=6$

Wenger, E., McDermott, R., \& Snyder, W. M. (2002). Seven Principles for cultivating Communities of Practice. In Cultivating Communites of Practice: A Guide to Managing Knowledge (pp. 4964). EE. UU.: Harvard Business School Press. 